\title{
The swollen leg: is it deep vein thrombosis? The experience of a tertiary referral center in Sri Lanka
}

\author{
S M Wijeyaratne', and A H Sheriffdeen ${ }^{2}$
}

(Index words: Swollen leg, deep vein thrombosis, risk factors, duplex scan)

\begin{abstract}
Introduction The role played by deep vein thrombosis (DVT) as a cause of leg swelling in Sri Lanka is unknown.

Purpose To study the prevalence of proximal DVT, value of risk factors and clinical features and attitudes of clinicians towards diagnosis and treatment of DVT among those presenting with leg swelling as the main complaint.
\end{abstract}

Design Prospective consecutive referrals to the University Surgical Unit, Colombo, over 10 months starting in January 1998.

Inclusion criteria DVT suspects with swollen legs.

Exclusion criteria Varicose veins, leg ulceration and generalized oedema.

Methods Color duplex scanning of the proximal deep veins and the superficial veins to detect occlusion and reflux. Information on previous diagnoses and treatment were obtained from the medical records.

Results $63 / 137$ (45\%) had deep venous causes for oedema. Fever with rigors and inguinal lymphaedenopathy were strongly predictive of non DVT causes. Filariasis is the first diagnosis and DVT is considered late among the outpatients.

Conclusions DVT is a common cause of leg swelling among those referred.

\section{Introduction}

Deep vein thrombosis (DVT) is an important cause of leg swelling which is thought to be rare in Sri Lanka. The only previous report on DVT was from Peradeniya in 1986, where they failed to detect DVT in a small group of prostatectomised patients (1). Morbidity from DVT includes pain, swelling and ulceration, which are easily appreciated while deaths from pulmonary embolism may not be realised $(2,3)$. These sequelae could be prevented or minimized by early diagnosis and anticoagulation (4). Therefore knowledge of the current situation regarding DVT in Sri Lanka with respect to one of its common presentations, the swollen leg, is important. We studied the prevalence of DVT, the value of risk factors and clinical features in its diagnosis, and the clinical approach of clinicians towards diagnosis and treatment of the swollen leg from among patients presenting with leg swelling as the main complaint.

\section{Methods}

Design A prospective study of consecutive referrals from $1^{\text {sl J January }} 1998$ to $31^{\text {s }}$ October 1998.

Patients All patients referred to the Vascular research laboratory of the Faculty of Medicine, University of Colombo (National Hospital of Sri Lanka) with the main complaint of leg swelling were studied. They were subjected to a detailed interview, physical examination and review of all previous medical records including prescriptions.

Age, sex, parity, duration of swelling, the occurrence of fever with rigors, recent surgical and non surgical trauma, immobilization, pregnancy, estrogen therapy, collagen diseases, malignancy, previous diagnoses, investigations and treatment were documented. Examination was carried out to detect varicose veins, leg ulceration, acute inflammation and pitting edema. The presence of swelling was accepted if the skin was pitting and/or if the mid calf or thigh circumference was at least $2 \mathrm{~cm}$ greater than on the normal leg. Subjects whose main complaint was varicose veins and/or active leg ulceration were excluded, even if they had a swollen leg. Those with bilateral swelling due to cardiac, respiratory, renal or liver disorders were also excluded.

Evaluation of the venous system The gold standard investigation for DVT has been contrast venography. In view of the invasive nature and complications of venography, noninvasive colour duplex ultrasonography has replaced it as the first line investigation for the diagnosis of DVT worldwide. Colour duplex scans were performed with a 7.5/5.5 MHz linear array transducer, using a Hewlett-Packard sonos 1000 machine. The deep and superficial venous systems in both lower limbs from the external iliac veins down to the popliteal vein were examined for patency and reflux.

Patency was inferred if there was complete colour filling and compressibility of the lumen. Colour filling defects, intraluminal echoes and incompressibility was considered as evidence of intraluminal clots. The morphological assessment was supported by hemodynamic evidence. The phasic variation of Doppler flow with respiration was interpreted as confirming proximal (ileocaval) patency. The augmentation of flow with distal limb compression was interpreted as confirming distal (tibialpopliteal-superficial femoral) patency. Venous reflux was studied with the limb dependent. Reflux was defined as the

'Senior Lecturer, ${ }^{2}$ Professor and Head, Department of Surgery, Faculty of Medicine, University of Colombo. (Corresponding author SMW, email: mandika59@hotmail.com; Received 20 July 1999, revised version accepted 4 August 2001) 
presence of reversed flow of more than 0.5 seconds on release of calf compression distal to the segment of vein under study. These diagnostic criteria for the diagnosis of venous obstruction and reflux on colour duplex scanning have been validated by comparing with venography $(5,6,7)$ and we reported a sensitivity of $100 \%$ and specificity of $94.4 \%$ for colour duplex in the diagnosis of DVT in our laboratory (8).

\section{Results}

133 patients with 137 swollen legs satisfied the criteria for study. The mean age of patients was 45.4 years (SEM 3.2). 70 (53.8\%) were females. 63 of 137 (46\%) had leg swelling due to DVT; 52 of these had occlusive proximal deep venous disease and 11 had deep vein reflux with wall thickening suggestive of recanalisation.

4 other limbs had superficial venous reflux alone in the absence of varicose veins. 70 limbs had normal veins. Amongst the 129 subjects with an asymptomatic contralateral leg, 8 had long saphenous vein (LSV) reflux and one had superficial femoral vein (SFV) reflux. 22/63 (35\%) had extensive thrombosis involving ileo-femoro-popliteal segments and the remainder was equally distributed in the ileo-femoral, femoro-popliteal and popliteal segments.

Analysis of clinicial features of the 137 limbs revealed that $55(40.1 \%)$ had fever with rigors, $41(29.9 \%)$ had painful regional lymphadenopathy, $126(89.8 \%)$ had leg pain and $48(35 \%)$ had identifiable risk factors for DVT. The sensitivity and specificity of each criterion was analyzed (Table 1). Known risk factors for DVT were observed in $40 / 63(63.4 \%)$ of patients with proven thromboses. These risk factors were more frequent among in-patients [22/32 (69\%)] than outpatients [18/105 (17\%)].

Table 1. Analysis of clinical presentation .

$$
\text { DVT Positive }
$$

DVT Negative

$\begin{array}{lll}\text { Rigor }+ & 03 & 52 \\ \text { Rigor - } & 60 & 22\end{array}$

Sensitivity $70.27 \%$, specificity $95.23 \%, P P V=94.545 \%$, $N P V=73.17 \%$ for non $D V T$ swelling

$\begin{array}{lll}\text { Lymph nodes + } & 06 & 35 \\ \text { Lymph nodes - } & 57 & 39\end{array}$

Sensitivity $47.29 \%$, specificity $90.47 \%, P P V=85.36 \%$, NPV $=59.37 \%$ for non DVT swelling

$\begin{array}{lll}\text { Pain + } & 59 & 64 \\ \text { Pain - } & 04 & 10\end{array}$

Sensitivity $93.65 \%$, specificity $13.51 \%, P P V=47.96 \%$, $N P V=71.42 \%$ for non $D V T$ swelling

$\begin{array}{lll}\text { Risk factors }+ & 40 & 08 \\ \text { Risk factors - } & 23 & 66\end{array}$

Relative risk for $D V T=14.3$
The initial diagnosis, investigation and treatment of the swollen leg:

\section{Initial diagnosis}

In-patient referrals accounted for 32 of 137 (23.4\%) swollen legs. DVT was the initial diagnosis in 30 (93.7\%) of them. 22 of the 30 (73.3\%) were positive for DVT. None of the inpatients with DVT had been misdiagnosed. Outpatient referrals accounted for 105 of $137(76.6 \%)$ swollen limbs. An initial diagnosis of DVT was made in 08 (7.6\%). DVT was confirmed in 41 (39\%) of the out-patients. Among those confirmed to have DVT, 03 had been diagnosed accurately at initial presentation and $38(92.6 \%)$ had other diagnostic considerations.

\section{Initial investigations performed}

$30 / 32(94 \%)$ of hospitalised patients were referred for duplex assessment prior to other investigations. In contrast 38/41 (93\%) of DVT positive out-patient referrals had filarial serology and/or white cell counts as the first investigation, and only 3 patients who were suspected of having DVT had urgent referral for imaging. Results of filarial serology (FAT) appear similar in those with and without DVT (Table 2). Initial treatment reflected the pattern of investigation and over $90 \%$ of the out-patients had completed a course of antifilarial and or antimicrobial therapy, $\mathbf{7 2 \%}$ of them combined with diuretics and analgesics.

Table 2. Filarial serology (expressed as percentages)

$\begin{array}{lcc} & D v t+ & D v t- \\ \text { Negative } & 22 & 20 \\ \text { Weakly+ve } & 18 & 22 \\ \text { Positive } & 01 & 04 \\ \text { Strongly+ve } & 00 & 02\end{array}$

\section{Discussion}

Swelling of the leg is a common clinical problem with many possible causes. Filarial oedema as a cause is of primary diagnostic concern among many medical practitioners in view of its endemicity in tropical countries like Sri Lanka. The other cause commonly considered is soft tissue bacterial infections. The contribution from DVT to leg swelling is unkown and is believed to be an insignificant problem, judging by the lack of reports on the subject in Sri Lanka.

Our data demonstrates that DVT is also an important cause of leg swelling in Sri Lanka with a prevalence of $45 \%$ among those referred to our specialist center. This is the first report addressing the problem of DVT in Sri Lanka. The high prevalence of DVT in this cohort can be explained on the basis that referrals were a selected sample of DVT suspects from among those with swollen legs presenting 
to the physician in the hospital and the community, and therefore would not reflect its true prevalence in the community. The positive rate for DVT amongst referrals to other vascular laboratories in general vary between 10 and $50 \%$, and ours is at the high end of the scale (9), which reflects a referral bias among those who refer. Failed initial treatment and worsening symptoms inspired most referrals. Gross symptoms and extensive thrombosis viz., lleo-femoral and popliteal thrombosis, in $35 \%$ at the time of referral reflects the high selectivity and threshold for referral. Hence it justifies the assumption that less severe symptoms due to DVT must be escaping early referral, and may be totally missed if they cause sudden death from pulmonary embolism or become apparent much later when it causes chronic leg ulceration. Although the high selectivity in referral practice would help maintain the workload of vascular laboratories at manageable levels, it would not benefit those who are diagnosed late or never. The high referral rates to vascular laboratories in the West have prompted research into finding cheaper and simpler screening methods for DVT such as d-dimer levels in blood (9).

With regard to clinical features studied, the presence of fever with rigors and painful inguinal masses at the onset has a high specificity and positive predictive value for non-DVT swellings. The same cannot be said for leg pain. Therefore a good history and examination would be usuful in selecting those that are more likely to have DVT. Recognized risk factors for DVT were associated with a high relative risk comparable to that previously reported in the West (11). Prevention is of extreme importance in those at high risk. This series consisted of $40 / 63(63.5 \%)$ such patients. Further research is necessary to identity "high risk" groups and to determine the prevalence of DVT among these groups in Sri Lanka, with a view to formulating protocols for thromboprophylaxis. 23/41 (56.9\%) of out-patients had no identifiable risk factors. Genetically determined natural anticoagulant deficiency states and acquired autoimmune conditions are the most likely causes in this group and needs further study.

This data depicts current practices in the management of the swollen leg in Sri Lanka. 41/105 (39\%) outpatients had DVT with $92.6 \%$ of them being initially misdiagnosed. Most swollen legs including those with DVT are initially and often repeatedly treated for filarial lymphoedema despite the FAT not supporting such a practice. With regards to FAT, only the different grades of positivity/ negativity were available for analysis. Neither the laboratory test method used, antibody titres nor its sensitivity and specificity in the diagnosis of filariasis are known. The pupose of this study was not to determine the filarial antibody status of these patients, but to highlight the popular diagnostic algorithm for a swollen leg in Sri Lanka. The obsession with filariasis was further highlighted, when even those with an established diagnosis of DVT at other centers when referred to our unit for reconfirmation had been requested to perform FAT test and take a course of diethycarbamazine. Awareness with regards to DVT as a cause of leg swelling seems satisfactory within the National Hospital environment. This may be due to the special interest in vascular diseases in our unit at the National Hospital and the availability of a 24-hour vascular service.

In conclusion this study has demonstrated that DVT occurred in $45 \%$ of consecutive referrals with leg swelling in Sri Lanka. Although these results do not reflect the prevalence of DVT among swollen legs in general, it does point to DVT being considered more as an afterthought rather than one that needs to be actively excluded early. The emphasis on filariasis as the cause of leg swelling seems excessive and these findings warrant a change in the approach to the management of the swollen leg in Sri Lanka. The drawbacks related to diagnosis could be corrected by an increased awareness of the value of certain clinical features, the relevance of risk factors, and better utilization of available ultrasound equipment. Further research needs to estimate the prevalence of DVT among high-risk groups with a view to adopting strategies for prophylaxis. Finally, more than half the thromboses among the outpatients were spontaneous; thus genetic and autoimmune causes need to be researched.

\section{References}

1. Sarathkumara KA, Ratnasena BGN, Herath KB, Ratnatunge PCA. Incidence of Post-operative Deep Vein Thrombosis in Sri Lankan patients (Preliminary Report), Proceedings of the Kandy Society of Medicine 1986; 9: 31

2. Dalen JE, Alpert JS. Natural history of pulmonary embolism. Progress in Cardiovascular Disease 1975; 17: 257-70.

3. Strandness DE Jr, Langlois YE, Cramer ME, Randlett A, Thiele BL. Long-term sequelae of acute venous thrombosis. Journal of the American Medical Association 1983; 250: 1289-92.

4. Goldhaber SZ, Morpurgo M. Diagnosis, treatment, and prevention of pulmonary embolism: report of the WHO/International Society and Federation of Cardiology Task Force. Journal of the American Medical Association 1992; 268: 1727-33.

5. White RH, McGahan JP, Daschbach MM, Hartling RP, et al. Diagnosis of deep vein thrombosis using duplex ultrasound. Annals of Internal Medicine 1989; 111: 297-304.

6. Lensing AWA, Prandoni P, Brandjes D, et al. Detection of deep vein thrombosis by real-time B-mode ultrasonography. New England Joumal of Medicine 1989; 320: 342-45.

7. Cogo A, Lensing AW, Wells $P$, et al. Noninvasive objective tests for the diagnosis of clinically suspected deep-vein thrombosis. Haemostasis 1995; 25: 27-39.

8. Wijeyaratne SM, Sheriffdeen AH. The value of colour duplex in suspected proximal deep vein thrombosis of the leg. Proceedings of the Annual Sessions of the College of Surgeons of Sri Lanka, July, 1999.

9. Harris LM, Curl GR, Booth FV, Hassett JM, Leney G, Ricotta JJ. Screening for asymptomatic deep vein thrombosis in surgical intensive care patients. Journal of Vascular Surgery 1997; 26: 764-9.

10. Lippi G, Mengoni A, Manzato F. Plasma D-dimer in the diagnosis of deep vein thrombosis Joumal of the American Medical Association 1998; 280: 1828-9.

11. Anderson FA, Wheeler HB. Venous thromboembolism: risk factors and prophylaxis. Clinics in Chest Medicine 1995; 16: 23551. 